\title{
On Block-wise Symmetric Signatures for Matchgates
}

\author{
Jin-Yi Cai ${ }^{1}$ and Pinyan $\mathrm{Lu}^{2 \star}$ \\ 1 Computer Sciences Department, University of Wisconsin \\ Madison, WI 53706, USA \\ jyc@cs.wisc.edu \\ 2 Department of Computer Science and Technology, Tsinghua University \\ Beijing, 100084, P. R. China \\ lpy@mails.tsinghua.edu.cn
}

\begin{abstract}
We give a classification of block-wise symmetric signatures in the theory of matchgate computations. The main proof technique is matchgate identities, a.k.a. useful Grassmann-Plücker identities.
\end{abstract}

\section{Introduction}

The most fundamental question in computational complexity theory is what differentiate between polynomial time and exponential time problems. On the one hand, we have many completeness results and conjectured separations of complexity classes. On the other hand we have precious few unconditional separations. In fact, the most spectacular advances in the field in the past 20 years have been upper bounds, i.e., surprising ways to do computation efficiently. Valiant's theory of matchgate and holographic algorithms $[10,12]$ is one such methodology.

The basic idea in matchgate computations is to encode $0-1$ bits of a computation in terms of perfect matchings. The complexity of graph matching is very interesting in its own right, having inspired the notion of $\mathrm{P}$ in the first place [4]. While a brute force attempt at graph matching seems to take exponential time, it turns out that the decision problem is in P. More relevant, counting perfect matchings is known to be in $\mathrm{P}$ for planar graphs by the FKT method $[6,7,9]$. (Counting all, not necessarily perfect, matchings for planar graphs is \#P-complete, as is counting perfect matchings for general graphs [5].) So one can say that graph matching is right at the border of polynomial time and (probably) exponential time. Valiant's theory of matchgate computations uses the FKT method as the starting point.

To give a flavor of this methodology, let's consider the problem $\#_{7} \mathrm{Pl}-\mathrm{R} t w-$ Mon-3CNF. Given a planar read-twice monotone 3CNF formula, this problem asks for the number of satisfying assignments modulo 7. Without the modulo 7,

\footnotetext{
* Supported by NSF CCR-0511679 and by the National Natural Science Foundation of China Grant 60553001 and the National Basic Research Program of China Grant 2007CB807900, 2007CB807901.
} 
it is \#P-complete even for such restricted formulae [13]. Furthermore, counting mod 2, denoted as \# ${ }_{2} \mathrm{Pl}$-Rtw-Mon-3CNF, is $\oplus \mathrm{P}$-complete (hence NP-hard). But, using matchgates Valiant showed that $\#_{7} \mathrm{Pl}-\mathrm{Rtw}-\mathrm{Mon}-3 \mathrm{CNF} \in \mathrm{P}$ [13].

A matchgate is a weighted planar graph with some external nodes. E.g., let $\pi$ be a path of length 3 : all 3 edges have weight 1 , and the two end vertices are external nodes. If we remove exactly one of the two external nodes we have 3 vertices left and therefore there is no perfect matching. If we remove either both or none of the two external nodes we get a unique perfect matching with weight 1 (the product of weights of matching edges). We can record this information as $(1,0,0,1)^{\mathrm{T}}$, indexed by $00,01,10,11$; this is called the (standard) signature of $\pi$. One can use this gadget to replace a Boolean variable $x$ in a planar formula $\varphi$, and $00,01,10,11$ will naturally correspond to truth values of $x$ to be fannedout to the 2 clauses of $\varphi$ in which $x$ appears (recall it is read-twice). Then the signature $(1,0,0,1)^{\mathrm{T}}$ indicates consistency of this truth assignment on $x$.

Now for each clause in $\varphi$ we wish to find a matchgate with 3 external nodes having signature $(0,1,1,1,1,1,1,1)^{\mathrm{T}}$, indexed by $000,001, \ldots, 111$. This signature corresponds to a Boolean OR. One can replace each clause by such a gadget, and connect its 3 external nodes to the gadgets of its 3 variables. Then the total number of perfect matchings of the resulting planar graph is exactly the number of satisfying assignments of $\varphi$. This can be computed by the FKT method, which would imply $\mathrm{P}^{\# \mathrm{P}}=\mathrm{P}$.

It turns out that a matchgate with the standard signature $(0,1,1,1,1,1,1,1)^{\mathrm{T}}$ does not exist. However, using a basis transformation a (non-standard) signature in the form $(0,1,1,1,1,1,1,1)^{\mathrm{T}}$ is realizable over the field $\mathbf{Z}_{7}$ (but not $\mathbf{Q}$ ). This gives the result that $\#_{7} \mathrm{Pl}-\mathrm{Rtw}-\mathrm{Mon}-3 \mathrm{CNF} \in \mathrm{P}$. (In this paper we will not be concerned with non-standard signatures.)

The signatures $(1,0,0,1)^{\mathrm{T}}$ and $(0,1,1,1,1,1,1,1)^{\mathrm{T}}$ are called symmetric signatures, since their values only depend on the Hamming weight of the index. Symmetric signatures have natural combinatorial meanings (such as two equal bits or the Boolean OR). Therefore the study of symmetric signatures is of foremost importance in order to understand the power of these exotic algorithms. To this end, we have achieved a complete classification of bit-wise symmetric signatures [?].

In Valiant's surprising algorithm for $\#_{7} \mathrm{Pl}-\mathrm{Rtw}-\mathrm{Mon}-3 \mathrm{CNF}$ he took another innovative step in the use of matchgates. In his algorithm, the matchgates have external nodes grouped in blocks of 2 each (called "2-rail" in [13]). This naturally raises the question of classification of block-wise symmetric signatures. This paper is concerned with this classification.

The classification theorem of block-wise symmetric signatures is more difficult compared to that of bit-wise symmetric signatures. The main reason for this is that matchgate signatures are characterized by a set of parity requirements (due to consideration of perfect matchings) and an exponential sized set of algebraic constraints called Matchgate Identities (MGI) a.k.a. the useful GrassmanPlücker Identities $[8,11,2,1]$. These MGI are non-linear, and are more subtle compared to parity requirements. They come about due to an equivalence 
between the perfect matching polynomial PerfMatch and the Pfaffian $[2,1]$. For bit-wise symmetric signatures, these MGI degenerate into something more readily treatable. This paper is the first time one is able to mount a successful and systematic attack on these MGI. We find proofs on MGI technically challenging, with almost every step a struggle (at least to the authors).

At a higher level, the new theory of matchgate and holographic algorithms represents a novel algorithm design methodology by Valiant, with its ultimate reach unknown. Will the new theory lead to a collapse of complexity classes? We don't know. Only a systematic study will (hopefully) tell. To get a classification theorem for block-wise symmetric signatures seems a useful step.

\section{Background}

Let $G=(V, E, W)$ be a weighted undirected planar graph. A matchgate $\Gamma$ is a tuple $(G, X)$ where $X \subseteq V$ is a set of external nodes, ordered counterclockwise on the external face. $\Gamma$ is called an odd (resp. even) matchgate if it has an odd (resp. even) number of nodes.

Each matchgate $\Gamma$ with $n$ external nodes is assigned a (standard) signature $\left(\Gamma^{\alpha}\right)_{\alpha \in\{0,1\}^{n}}$ with $2^{n}$ entries,

$$
\Gamma^{i_{1} i_{2} \ldots i_{n}}=\operatorname{PerfMatch}(G-Z)=\sum_{M} \prod_{(i, j) \in M} w_{i j},
$$

where the sum is over all perfect matchings $M$ of $G-Z$, and $Z \subseteq X$ is the subset of external nodes having the characteristic sequence $\chi_{Z}=i_{1} i_{2} \ldots i_{n}$.

An entry $\Gamma^{\alpha}$ is called an even (resp. odd) entry if the Hamming weight $\operatorname{wt}(\alpha)$ is even (resp. odd). It was proved in $[1,2]$ that standard signatures are characterized by the following two sets of conditions. (1) The parity requirements: either all even entries are 0 or all odd entries are 0 . This is due to perfect matchings. (2) A set of Matchgate Identities (MGI) defined as follows: A pattern $\alpha$ is an $n$-bit string, i.e., $\alpha \in\{0,1\}^{n}$. A position vector $P=\left\{p_{i}\right\}, i \in[l]$, is a subsequence of $\{1,2, \ldots, n\}$, i.e., $p_{i} \in[n]$ and $p_{1}<p_{2}<\cdots<p_{l}$. We also use $p$ to denote the pattern, whose $\left(p_{1}, p_{2}, \ldots, p_{l}\right)$-th bits are 1 and others are 0 . Let $e_{i} \in\{0,1\}^{n}$ be the pattern with 1 in the $i$-th bit and 0 elsewhere. Let $\alpha+\beta$ be the bitwise XOR of $\alpha$ and $\beta$. Then for any pattern $\alpha \in\{0,1\}^{n}$ and any position vector $P=\left\{p_{i}\right\}, i \in[l]$,

$$
\sum_{i=1}^{l}(-1)^{i} \Gamma^{\alpha+e_{p_{i}}} \Gamma^{\alpha+p+e_{p_{i}}}=0 .
$$

The use of MGI will be central in this paper. These MGI come from the Grassmann-Plücker identities valid for Pfaffians. In fact initially Valiant introduced two theories of matchgate computation: The first is the matchcircuit theory with general (non-planar) matchgates [10]. These matchgates have characters which are defined in terms of Pfaffians. The second is the theory 
of matchgrid/holographic algorithms [12]. These use planar matchgates with signatures defined by PerfMatch. In [2] it was proved that MGI characterize (general) matchgate characters. In [1] an equivalence theorem of characters and signatures was established, and thus MGI also characterize planar matchgate signatures. The dual forms of the theory have been useful in both ways: some times it is easier to reason and construct planar gadgets, other times the algebraic Pfaffian setup seems essential. A case in point is symmetric signatures.

A signature $\Gamma$ is (bit-wise) symmetric if $\Gamma^{\alpha}$ only depends on wt $(\alpha)$. A bitwise symmetric signature can be denoted as $\left[z_{0}, z_{1}, \ldots, z_{n}\right]$, where $\Gamma^{\alpha}=z_{\mathrm{wt}(\alpha)}$. It was proved in [2] that for even matchgates, a signature $\left[z_{0}, z_{1}, \ldots, z_{n}\right]$ is realizable iff for all odd $i, z_{i}=0$, and there exist constants $r_{1}, r_{2}$ and $\lambda$, such that $z_{2 i}=\lambda \cdot\left(r_{1}\right)^{\lfloor n / 2\rfloor-i} \cdot\left(r_{2}\right)^{i}$, for $0 \leq i \leq\left\lfloor\frac{n}{2}\right\rfloor$. Similar results hold for odd matchgates. These are proved via MGI and Pfaffians. It is interesting to note that the only construction for a planar matchgate realizing this signature is through a non-planar matchgate $\Gamma$ and its character theory. There is no known direct construction.

A tensor $\left(\Gamma^{\alpha}\right)$ on index $\alpha=\alpha_{1} \ldots \alpha_{n}$, where each $\alpha_{i} \in\{0,1\}^{k}$, is blockwise symmetric if $\Gamma^{\alpha}$ only depends on the number of $k$-bit patterns of $\alpha_{i}$, i.e., $\Gamma^{\cdots \alpha_{i} \ldots \alpha_{j} \ldots}=\Gamma^{\cdots \alpha_{j} \ldots \alpha_{i} \cdots}$, for all $1 \leq i<j \leq n$.

For an even (resp. odd) matchgate $\Gamma$ with arity $n$, the condensed signature $\left(g^{\alpha}\right)$ of $\Gamma$ is a tensor of arity $n-1$, and $g^{\alpha}=\Gamma^{\alpha b}$ (resp. $\left.g^{\alpha}=\Gamma^{\alpha \bar{b}}\right)$, where $\alpha \in\{0,1\}^{n-1}$ and $b=p(\alpha)$ is the parity of $\operatorname{wt}(\alpha)$.

\section{Decomposition Theory for Block-wise Symmetric Signatures}

Theorem 1. Let $\left(\Gamma^{\alpha}\right)$ be a block-wise symmetric tensor with block size $k$ and arity $n k$. Assume $n \geq 4$ and $\Gamma^{00 \cdots 0} \neq 0$. Then $\Gamma$ is realizable by a matchgate iff there exist a matchgate $\Gamma_{0}$ with arity $k+1$ and condensed signature $\left(g^{\alpha}\right)_{\alpha \in\{0,1\}^{k}}$, and a symmetric matchgate $\Gamma_{s}$ such that

$$
\Gamma^{\alpha_{1} \alpha_{2} \cdots \alpha_{n}}=\Gamma_{s}^{p\left(\alpha_{1}\right) p\left(\alpha_{2}\right) \cdots p\left(\alpha_{n}\right)} g^{\alpha_{1}} g^{\alpha_{2}} \cdots g^{\alpha_{n}} .
$$

Proof: We prove " $\Leftarrow$ " by a direct construction. In Figure 1, we extend every external node of $\Gamma_{s}$ by a copy of the matchgate with condensed signature $g$, and view the remaining $k$ external nodes of each copy as external. This gives us a new matchgate with $n k$ external nodes, whose signature is given by (2). Therefore every signature which has form (2) is realizable.

Now we prove " $\Rightarrow$ ": Since $\Gamma^{00 \cdots 0} \neq 0$, by adding an extra isolated edge with weight $1 / \Gamma^{00 \cdots 0}$ we can assume $\Gamma^{00 \cdots 0}=1$. First we assume $r_{1}=\Gamma^{e_{1} e_{1} 00 \cdots 0} \neq 0$ (where for convenience we consider $e_{1} \in\{0,1\}^{k}$ ), and prove the theorem under this assumption. We take $\Gamma_{s}$ to be an even symmetric matchgate with signature $z_{2 i}=\left(r_{1}\right)^{-i}$. By [2] this $\Gamma_{s}$ exists. Since the given $\left(\Gamma^{\alpha}\right)$ is realizable, it can be realized by a matchgate $\Gamma$ with $n k$ external nodes. View its first $k+1$ external nodes still as external nodes and the other nodes as internal, we have a matchgate 


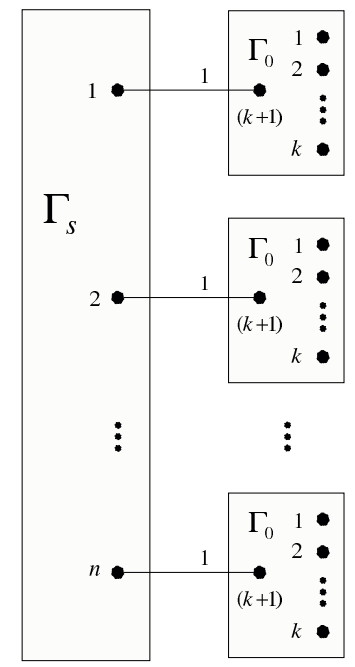

Fig. 1. Block-wise symmetric signature

with $k+1$ external nodes. This is our $\Gamma_{0}$. By definition its condensed signature is

$$
g^{\alpha}=\left\{\begin{array}{cc}
\Gamma^{\alpha 00 \cdots 0} & \text { when wt }(\alpha) \text { is even, } \\
\Gamma^{\alpha e_{1} 0 \cdots 0} & \text { when } \operatorname{wt}(\alpha) \text { is odd }
\end{array}\right.
$$

Note that $g^{0}=1$ and $g^{e_{1}}=r_{1}$. We prove (2) by induction on $\operatorname{wt}\left(\alpha_{1} \alpha_{2} \cdots \alpha_{n}\right) \geq 0$ and $\operatorname{wt}\left(\alpha_{1} \alpha_{2} \cdots \alpha_{n}\right)$ is even.

If $\operatorname{wt}\left(\alpha_{1} \alpha_{2} \cdots \alpha_{n}\right)=0$, we have the only case that $\alpha_{1} \alpha_{2} \cdots \alpha_{n}=00 \cdots 0$. In this case (2) is obvious.

If wt $\left(\alpha_{1} \alpha_{2} \cdots \alpha_{n}\right)=2$, we have two cases depending on whether the two 1s are in the same block or not. If they are in the same block, we can assume it is in the first block since $\Gamma$ is block symmetric, then $\Gamma^{\alpha_{1} \alpha_{2} \cdots \alpha_{n}}=\Gamma^{\alpha_{1} 00 \cdots 0}=g^{\alpha_{1}}$ and (2) is satisfied. If they are not in the same block, by symmetry, we may assume $\alpha_{1} \alpha_{2} \cdots \alpha_{n}$ has the form $e_{i} e_{j} 00 \cdots 0$. When 0 appears in the sup index of $\Gamma$, sup index of $g$, a pattern or positions used by a MGI for $\Gamma$, it means a block of all zero. Using the pattern $0 e_{j} e_{1} e_{1} 00 \cdots 0$ and positions $e_{i} e_{j} e_{1} e_{1} 00 \cdots 0$, from (1) we have the following matchgate identity (applying block-wise symmetry):

$\Gamma^{e_{i} e_{j} e_{1} e_{1} 0 \cdots 0} \Gamma^{00 \cdots 0}-\Gamma^{e_{1} e_{1} 0 \cdots 0} \Gamma^{e_{i} e_{j} 0 \cdots 0}+\Gamma^{e_{j} e_{1} 0 \cdots 0} \Gamma^{e_{i} e_{1} 0 \cdots 0}-\Gamma^{e_{j} e_{1} 0 \cdots 0} \Gamma^{e_{i} e_{1} 0 \cdots 0}=0$.

The last two terms cancel out, we get:

$$
\Gamma^{e_{i} e_{j} e_{1} e_{1} 00 \cdots 0}=\Gamma^{e_{i} e_{j} 00 \cdots 0} \Gamma^{e_{1} e_{1} 00 \cdots 0} .
$$

Next, using the pattern $0 e_{1} e_{j} e_{1} 00 \cdots 0$ and positions $e_{i} e_{1} e_{j} e_{1} 00 \cdots 0$, we have the following matchgate identity:

$\Gamma^{e_{i} e_{j} e_{1} e_{1} 0 \cdots 0} \Gamma^{00 \cdots 0}-\Gamma^{e_{j} e_{1} 0 \cdots 0} \Gamma^{e_{i} e_{1} 0 \cdots 0}+\Gamma^{e_{1} e_{1} 0 \cdots 0} \Gamma^{e_{i} e_{j} 0 \cdots 0}-\Gamma^{e_{j} e_{1} 0 \cdots 0} \Gamma^{e_{i} e_{1} 0 \cdots 0}=0$. 
Together with (3), we have $\Gamma^{e_{i} e_{j} 00 \cdots 0} \Gamma^{e_{1} e_{1} 00 \cdots 0}=\Gamma^{e_{i} e_{1} 00 \cdots 0} \Gamma^{e_{j} e_{1} 00 \cdots 0}$. Since $\Gamma^{e_{1} e_{1} 00 \cdots 0}=r_{1} \neq 0$, we have $\Gamma^{e_{i} e_{j} 00 \cdots 0}=\Gamma^{e_{i} e_{1} 00 \cdots 0} \Gamma^{e_{j} e_{1} 00 \cdots 0} / r_{1}=\left(r_{1}\right)^{-1} g^{e_{i}} g^{e_{j}}$. So (2) is satisfied.

Inductively we assume (2) has been proved for all wt $\left(\alpha_{1} \alpha_{2} \cdots \alpha_{n}\right) \leq 2(i-1)$, for some $i \geq 2$. Now wt $\left(\alpha_{1} \alpha_{2} \cdots \alpha_{n}\right)=2 i>0$. By symmetry, we can assume $\alpha_{1} \neq$ $00 \cdots 0$. Let $t$ be the position of the first 1 in $\alpha_{1}$. Using the pattern $\alpha_{1} \alpha_{2} \cdots \alpha_{n}+e_{t}$ and positions $\alpha_{1} \alpha_{2} \cdots \alpha_{n}$ (we denote it as $P=\left\{p_{j}\right\}$ where $j=1,2, \ldots, 2 i$ ), we have the following matchgate identity:

$$
\Gamma^{\alpha_{1} \alpha_{2} \cdots \alpha_{n}}=\sum_{j=2}^{2 i}(-1)^{j} \Gamma^{\alpha_{1} \alpha_{2} \cdots \alpha_{n}+e_{t}+e_{p_{j}}} \Gamma^{e_{t}+e_{p_{j}}} .
$$

Since every $\Gamma^{\beta}$ in the RHS has $\operatorname{wt}(\beta) \leq 2 i-2$, we can apply (2) to them.

Now we do the summation of the RHS in (4) block by block; the sum of the $r$-th block is denoted as $S_{r}$. Let $w_{r}=\operatorname{wt}\left(\alpha_{r}\right)$. Let $2 q$ be the number of odd $w_{r}$, i.e., the number of blocks among $\alpha_{1}, \alpha_{2}, \ldots, \alpha_{n}$ with odd weight. Note that this number is even.

For the first block, if $w_{1}=1$, then $S_{1}=0$, being an empty sum. Assume $w_{1}>1$. In the notation below we consider $e_{t}, e_{p_{j}} \in\{0,1\}^{k}$ for convenience.

$$
\begin{aligned}
S_{1} & =\sum_{j=2}^{w_{1}}(-1)^{j} \Gamma^{\left(\alpha_{1}+e_{t}+e_{p_{j}}\right) \alpha_{2} \cdots \alpha_{n}} \Gamma^{\left(e_{t}+e_{p_{j}}\right) 00 \cdots 0} \\
& =r_{1}^{-q} g^{\alpha_{2}} \cdots g^{\alpha_{n}} \sum_{j=2}^{w_{1}}(-1)^{j} g^{\alpha_{1}+e_{t}+e_{p_{j}}} g^{e_{t}+e_{p_{j}}} .
\end{aligned}
$$

Note that the exponent $q$ in $r_{1}^{-q}$ comes from the fact that the number of blocks with odd weight among $\alpha_{1}+e_{t}+e_{p_{j}}, \alpha_{2}, \ldots, \alpha_{n}$ is $2 q$.

If $w_{1}$ is odd, using the pattern $\left(\alpha_{1}+e_{t}\right) 1$ and positions $\alpha_{1} 1$, we have the following matchgate identity for $\Gamma_{0}$ :

$$
-g^{\alpha_{1}}+\sum_{j=2}^{w_{1}}(-1)^{j} g^{\alpha_{1}+e_{t}+e_{p_{j}}} g^{e_{t}+e_{p_{j}}}+g^{\alpha_{1}+e_{t}} g^{e_{t}}=0 .
$$

Substituting this in (6), we have:

$$
S_{1}=r_{1}^{-q} g^{\alpha_{2}} \cdots g^{\alpha_{n}}\left(g^{\alpha_{1}}-g^{\alpha_{1}+e_{t}} g^{e_{t}}\right) .
$$

We note that this is also valid for $w_{1}=1$.

If $w_{1}$ is even, using the pattern $\left(\alpha_{1}+e_{t}\right) 0$ and positions $\alpha_{1} 0$, we have the following matchgate identity for $\Gamma_{0}$ :

$$
-g^{\alpha_{1}}+\sum_{j=2}^{w_{1}}(-1)^{j} g^{\alpha_{1}+e_{t}+e_{p_{j}}} g^{e_{t}+e_{p_{j}}}=0 .
$$


Substituting this in (6), we have:

$$
S_{1}=r_{1}^{-q} g^{\alpha_{1}} g^{\alpha_{2}} \cdots g^{\alpha_{n}} .
$$

If all $S_{r}$ are empty block-wise sums for $r>1$ (i.e., $w_{r}=0$ for all $r>1$ ), then $w_{1}$ must be even, and we are done. Now suppose there are non-empty block-wise sums $S_{r}$, for $r>1$. For the $r$-th block, let $v_{r}$ be the number of $1 \mathrm{~s}$ in the first $r-1$ blocks, and $p_{j}^{r}\left(j \in\left[w_{r}\right]\right)$ be the position of the $j$-th 1 in $\alpha_{r}$. Then

$$
\begin{aligned}
S_{r} & =(-1)^{v_{r}} \sum_{j=1}^{w_{r}}(-1)^{j} \Gamma^{\left(\alpha_{1}+e_{t}\right) \alpha_{2} \cdots\left(\alpha_{r}+e_{p_{j}^{r}}\right) \cdots \alpha_{n}} \Gamma^{\left(e_{t}\right) 00 \cdots\left(e_{p_{j}^{r}}\right) \cdots 0} \\
& =(-1)^{v_{r}} r_{1}^{-q^{\prime}} g^{e_{t}} g^{\alpha_{1}+e_{t}} g^{\alpha_{2}} \cdots \widehat{g^{\alpha_{r}}} \cdots g^{\alpha_{n}} \sum_{j=1}^{w_{r}}(-1)^{j} g^{\alpha_{r}+e_{p_{j}^{r}}} g^{e_{p_{j}^{r}}^{r}},
\end{aligned}
$$

where $\widehat{g^{\alpha_{r}}}$ denotes a missing factor, and $2 q^{\prime}$ is the total number of odd blocks in $\alpha_{1}+e_{t}, \alpha_{2}, \ldots, \alpha_{r}+e_{p_{j}^{r}}, \ldots, \alpha_{n}$ from the first factor $\Gamma$ and in $\left(e_{t}\right) 00 \cdots\left(e_{p_{j}^{r}}\right) \cdots 0$ from the second factor $\Gamma$. If $w_{r}$ is even, using the pattern $\alpha_{r} 1$ and positions $\alpha_{r} 0$, we have the following matchgate identity for $\Gamma_{0}$ :

$$
\sum_{j=1}^{w_{r}}(-1)^{j} g^{\alpha_{r}+e_{p_{j}^{r}}} g^{e_{p_{j}^{r}}}=0 .
$$

Substituting this in (10), we have $S_{r}=0$.

Therefore, among block sums $S_{r}$, for $r>1$, we need only consider blocks with odd $w_{r}$. Assume $w_{r}$ is odd now, we have $q^{\prime}=q$ if $w_{1}$ is odd, and $q^{\prime}=q+1$ if $w_{1}$ is even. Using the pattern $\alpha_{r} 0$ and positions $\alpha_{r} 1$, we have the following MGI for $\Gamma_{0}$ :

$$
\sum_{j=1}^{w_{r}}(-1)^{j} g^{\alpha_{r}+e_{p_{j}^{r}}} g^{e_{p_{j}^{r}}}+g^{\alpha_{r}}=0 .
$$

Substituting this in (10), we have $S_{r}=-(-1)^{v_{r}} r_{1}^{-q^{\prime}} g^{e_{t}} g^{\alpha_{1}+e_{t}} g^{\alpha_{2}} \cdots g^{\alpha_{r}} \cdots g^{\alpha_{n}}$.

To summarize, after the first block sum $S_{1}$, every even block will be zero, and every odd block will alternatingly contribute a $\pm r_{1}^{-q^{\prime}} g^{e_{t}} g^{\alpha_{1}+e_{t}} g^{\alpha_{2}} \cdots g^{\alpha_{n}}$. If $S_{1}$ is an even block sum, then this alternating sum has an even number of such terms, and they all cancel out. This leaves us with the desired result $\Gamma^{\alpha_{1} \alpha_{2} \cdots \alpha_{n}}=$ $S_{1}=r_{1}^{-q} g^{\alpha_{1}} g^{\alpha_{2}} \cdots g^{\alpha_{n}}$ from (8). If the first block is odd, then $q^{\prime}=q$, and there are an odd number of alternating $S_{r}$ for $r>1$ and $w_{r}$ odd, starting with the sign $-(-1)^{v_{2}}=+1$. These will cancel out pairwise except one $r_{1}^{-q} g^{e_{t}} g^{\alpha_{1}+e_{t}} g^{\alpha_{2}} \cdots g^{\alpha_{n}}$ left, which cancels the $-r_{1}^{-q} g^{e_{t}} g^{\alpha_{1}+e_{t}} g^{\alpha_{2}} \cdots g^{\alpha_{n}}$ in $S_{1}$ from (7). Finally in either cases, we have $\Gamma^{\alpha_{1} \alpha_{2} \cdots \alpha_{n}}=r_{1}^{-q} g^{\alpha_{1}} g^{\alpha_{2}} \cdots g^{\alpha_{n}}$. This is precisely (2).

Now we consider the case $\Gamma^{e_{1} e_{1} 00 \cdots 0}=0$. If there exists any $i \in[k]$ such that $\Gamma^{e_{i} e_{i} 00 \cdots 0} \neq 0$, the above proof can go through similarly. Therefore we assume for all $i \in[k], \Gamma^{e_{i} e_{i} 00 \cdots 0}=0$. 
Consider any $1 \leq i, j, s, t \leq k$ (not necessarily distinct). Using the pattern $0 e_{j} e_{s} e_{t} 00 \cdots 0$ and positions $e_{i} e_{j} e_{s} e_{t} 00 \cdots 0$ we get (applying block symmetry),

$\Gamma^{e_{i} e_{j} e_{s} e_{t} 0 \cdots 0} \Gamma^{00 \cdots 0}-\Gamma^{e_{s} e_{t} 0 \cdots 0} \Gamma^{e_{i} e_{j} 0 \cdots 0}+\Gamma^{e_{i} e_{s} 0 \cdots 0} \Gamma^{e_{j} e_{t} 0 \cdots 0}-\Gamma^{e_{j} e_{s} 0 \cdots 0} \Gamma^{e_{i} e_{t} 0 \cdots 0}=0$.

Also use the pattern $0 e_{s} e_{j} e_{t} 00 \cdots 0$ and positions $e_{i} e_{s} e_{j} e_{t} 00 \cdots 0$ we get

$\Gamma^{e_{i} e_{s} e_{j} e_{t} 0 \cdots 0} \Gamma^{00 \cdots 0}-\Gamma^{e_{j} e_{t} 0 \cdots 0} \Gamma^{e_{i} e_{s} 0 \cdots 0}+\Gamma^{e_{s} e_{t} 0 \cdots 0} \Gamma^{e_{i} e_{j} 0 \cdots 0}-\Gamma^{e_{s} e_{j} 0 \cdots 0} \Gamma^{e_{i} e_{t} 0 \cdots 0}=0$.

Adding the two, we get $\Gamma^{e_{i} e_{s} e_{j} e_{t} 00 \cdots 0}=\Gamma^{e_{s} e_{j} 00 \cdots 0} \Gamma^{e_{i} e_{t} 00 \cdots 0}$.

From this we have

$$
\left(\Gamma^{e_{i} e_{j} 00 \cdots 0}\right)^{2}=\Gamma^{e_{i} e_{j} e_{i} e_{j} 00 \cdots 0}=\Gamma^{e_{i} e_{j} e_{j} e_{i} 00 \cdots 0}=\Gamma^{e_{i} e_{i} 00 \cdots 0} \Gamma^{e_{j} e_{j} 00 \cdots 0}=0 .
$$

Therefore for all $i, j \in[k]$, we have $\Gamma^{e_{i} e_{j} 00 \cdots 0}=0$. Now we define $g^{\alpha}=\Gamma^{\alpha 00 \cdots 0}$ when $\operatorname{wt}(\alpha)$ is even, and $g^{\alpha}=0$ when $\operatorname{wt}(\alpha)$ is odd, and inductively prove (2) similarly as before. $\left(g^{\alpha}\right)$ is the condensed signature of a realizable matchgate $\Gamma_{0}$ of arity $k+1$ obtained from $\Gamma$ as follows: View its first $k$ external nodes (in the first block) still as external and the rest as internal, add a new isolated edge with weight 1 , and one end as the $(k+1)$-st external node and the other end an internal node. We will still arrive at (4). Now all block sums $S_{r}=0$, for $r>1$, since it involves a $\Gamma^{e_{t}+e_{p_{j}}}$, and $e_{t}$ appears in the first block.

Consider the first block sum $S_{1}$. Suppose $q>0$, i.e., there are some odd $w_{r}$. Then there are at least two odd blocks. Only the first block has a changed index in the sum, so some odd block among $\alpha_{2}, \ldots, \alpha_{n}$ remains in $\Gamma^{\alpha_{1} \alpha_{2} \cdots \alpha_{n}+e_{t}+e_{p_{j}}}$. Thus, by induction it is 0 , since the corresponding $g^{\alpha_{i}}=0$. Now suppose $q=0$, i.e., all blocks are even. By induction we get

$$
\Gamma^{\alpha_{1} \alpha_{2} \cdots \alpha_{n}}=g^{\alpha_{2}} \cdots g^{\alpha_{n}} \sum_{j=2}^{w_{1}}(-1)^{j} g^{\alpha_{1}+e_{t}+e_{p_{j}}} g^{e_{t}+e_{p_{j}}}
$$

Using the pattern $\left(\alpha_{1}+e_{t}\right) 0$ and positions $\alpha_{1} 0$ on $\Gamma_{0}$, we have MGI,

$$
-g^{\alpha_{1}}+\sum_{j=2}^{w_{1}}(-1)^{j} g^{\alpha_{1}+e_{t}+e_{p_{j}}} g^{e_{t}+e_{p_{j}}}=0
$$

This gives $\Gamma^{\alpha_{1} \alpha_{2} \cdots \alpha_{n}}=g^{\alpha_{1}} g^{\alpha_{2}} \cdots g^{\alpha_{n}}$ proving (2).

In Theorem 1, we assumed $\Gamma^{00 \cdots 0} \neq 0$. So it must be an even matchgate. For odd matchgates, we have a similar theorem under the assumption $\Gamma^{e_{1} 00 \cdots 0} \neq$ 0 . This proof is slightly more complicated but along similar lines. Due to space limitations we present it in Appendix A. These theorems give an elegant decomposition structure of block-wise symmetric signatures. There is an underlying bit-wise symmetric signature $\Gamma_{s}$, whose structure is very clear to us. Therefore, the realizability condition is within each block. 


\section{Characterization of Block-wise Symmetric Signature with Block Size 2}

In Theorem 1 , we have two assumptions $n \geq 4$ and $\Gamma^{00 \ldots 0} \neq 0 . n \geq 4$ is necessary for some boundary reason. The assumption $\Gamma^{00 \ldots 0} \neq 0$ is more technical but we are not able to bypass it in general. However, in this section we show that this assumption is not necessary for block size $k=2$.

Theorem 2. If $\Gamma$ is a block-wise symmetric signature for some matchgate, whose block size is 2 and arity $2 n$ where $n \geq 4$. Then there exist four numbers $g^{00}, g^{01}, g^{10}, g^{11}$ and a realizable bit-wise symmetric signature $\Gamma_{s}$ such that

$$
\Gamma^{\alpha_{1} \alpha_{2} \cdots \alpha_{n}}=\Gamma_{s}^{p\left(\alpha_{1}\right) p\left(\alpha_{2}\right) \cdots p\left(\alpha_{n}\right)} g^{\alpha_{1}} g^{\alpha_{2}} \cdots g^{\alpha_{n}} .
$$

We only prove it for even matchgates here; the proof is similar for odd matchgates. If $\Gamma^{00,00, \ldots, 00} \neq 0$ or $\Gamma^{11,11, \ldots, 11} \neq 0$ (we use "," to separate blocks), we are done by Theorem 1 . Note that flipping all bits preserves block-symmetry. Now we assume $\Gamma$ is an even matchgate, $n \geq 4$, and $\Gamma^{00,00, \ldots, 00}=\Gamma^{11,11, \ldots, 11}=0$. This assumption is made for all the following Claims.

Claim 1 For any $\alpha \in\{00,01,10,11\}^{n-4}$, we have

$$
\begin{gathered}
\Gamma^{01,01,01,01, \alpha} \Gamma^{00,00,00,00, \alpha}=\left(\Gamma^{01,01,00,00, \alpha}\right)^{2} . \\
\Gamma^{10,10,10,10, \alpha} \Gamma^{00,00,00,00, \alpha}=\left(\Gamma^{10,10,00,00, \alpha}\right)^{2} . \\
\Gamma^{01,01,10,10, \alpha} \Gamma^{00,00,00,00, \alpha}=\Gamma^{01,01,00,00, \alpha} G^{10,10,00,00, \alpha}=\left(\Gamma^{01,10,00,00, \alpha}\right)^{2} .
\end{gathered}
$$

Proof: All three equations follow from MGI. The $\alpha$ part is not involved in the MGI. This means that the pattern for these bits is exactly $\alpha$ and the position vector bits for these bit locations are all 0 . For convenience, we only list below the pattern and positions for the other bits, which are really involved in the MGI. We also use this simplified notation in the following Claims.

This Claim is quite direct from MGI. We only list the pattern and positions used, and omit the actual MGI. The first equation uses the pattern 00, 01, 01,01 and positions $01,01,01,01$. The second equation uses the pattern $00,10,10,10$ and positions $10,10,10,10$. The last equation is from two MGI: one uses the pattern $00,01,10,10$ and positions $01,01,10,10$, the other uses the pattern $00,10,01,10$ and positions $01,10,01,10$.

\section{Claim 2}

$$
\begin{aligned}
& \Gamma^{00,00,\{00,01,10\}^{n-2}}=0 . \\
& \Gamma^{11,11,\{11,01,10\}^{n-2}}=0 .
\end{aligned}
$$

Proof: We only prove $\Gamma^{00,00,\{00,01,10\}^{n-2}}=0$; the second equation can be obtained for the first by flipping all the bits. For $\alpha \in\{00,01,10\}^{n-2}$, we prove it by induction on $\operatorname{wt}(\alpha) \geq 0$ and $\operatorname{wt}(\alpha)$ is even. The case $\operatorname{wt}(\alpha)=0$ is by assumption. We use Claim 1 to go from weight $i$ to weight $i+2$. 
Claim 3 For any $\alpha \in\{00,01,10,11\}^{n-3}$,

$$
\begin{aligned}
& \Gamma^{00,00,00, \alpha}=0 . \\
& \Gamma^{11,11,11, \alpha}=0 .
\end{aligned}
$$

Proof: We also only need to prove $\Gamma^{00,00,00, \alpha}=0$. For $\alpha \in\{00,01,10,11\}^{n-3}$, we prove it by induction on the number of non- "00" blocks in $\alpha$. (We denote this number by $N_{0}(\alpha)$.)

If every block in $\alpha$ is 00 , then it is by assumption. Inductively we assume it has been proved for all $N_{0}(\alpha)<i$. Now $N_{0}(\alpha)=i$. If $\alpha$ does not have any block "11", it has been proved by Claim 2. Otherwise, we can assume $\alpha=11, \alpha$ ' by block-symmetry. Since $N_{0}\left(00, \alpha^{\prime}\right)=i-1$, we have $\Gamma^{00,00,00,00, \alpha^{\prime}}=0$.

Using the pattern $00,00,01,11$ and positions $00,00,11,11$, we have MGI: (Note that we omit the $\alpha^{\prime}$ part, and also we omit the symbol $\Gamma$ in the MGI.)

$$
\begin{array}{r}
0=(00,00,11,11)(00,00,00,00) \\
-(00,00,00,11)(00,00,11,00) \\
+(00,00,01,01)(00,00,10,10) \\
-(00,00,01,10)(00,00,10,01)
\end{array}
$$

The first term is 0 , and by Claim 1 , the last two terms cancel out. It follows that $\Gamma^{00,00,00,11, \alpha^{\prime}} \Gamma^{00,00,11,00, \alpha^{\prime}}=0$, which is exactly $\Gamma^{00,00,00, \alpha}=0$.

From Claim 3 and Claim 1, we have

Claim 4 For any $\alpha \in\{00,01,10,11\}^{n-4}$,

$$
\Gamma^{01,10,00,00, \alpha}=\Gamma^{01,01,00,00, \alpha}=\Gamma^{10,10,00,00, \alpha}=0 .
$$

Claim 5 For any $\alpha \in\{00,01,10,11\}^{n-2}$, the following are all valid,

$$
\Gamma^{00,00, \alpha}=0, \quad \Gamma^{11,11, \alpha}=0, \quad \Gamma^{00,11, \alpha}=0 .
$$

Claim 5 says that every non-zero entry $\Gamma^{\alpha}$ can have at most one even block. This is an important step in the proof. However, due to space limitation, the proof is omitted here, and is presented in Appendix B. The proof is by repeated applications of MGI (death by a thousand cuts, an ancient Chinese disgrace; unfortunately we cannot find a coup de grâce.)

Claim 6 For any $\alpha \in\{00,01,10,11\}^{n-2}$, we have

$$
\Gamma^{01,01, \alpha} \Gamma^{10,10, \alpha}=\left(\Gamma^{01,10, \alpha}\right)^{2} .
$$

Proof: Using the pattern 00, 01 and positions 11,11 (omitting $\alpha$ ), we have MGI:

$$
0=(10,01)(01,10)-(01,01)(10,10)+(00,11)(11,00)-(00,00)(11,11) .
$$

From Claim 5, we know the last two terms are both 0. So we have

$$
\Gamma^{01,01, \alpha} \Gamma^{10,10, \alpha}=\left(\Gamma^{01,10, \alpha}\right)^{2} .
$$


Claim 7 For $n \geq 4, k=2$, if $n$ is even and $\Gamma^{00,00, \ldots, 00}=\Gamma^{11,11, \ldots, 11}=0$, Theorem 2 holds.

Proof: Suppose $\Gamma^{\alpha_{1}, \alpha_{2}, \ldots, \alpha_{n}} \neq 0$, we show each $\alpha_{i} \in\{01,10\}$. Since $n$ is even and we have an even matchgate, the number of odd blocks must be even, so that if it has any even block it has at least two even blocks. Then by Claim 5 it is 0 .

If $\Gamma^{01,01, \ldots, 01} \neq 0$, w.l.o.g, we assume $\Gamma^{01,01, \ldots, 01}=1$. Let $\Gamma_{s}$ be the matchgate having symmetric signature $[0,0, \ldots, 0,1]$ (in the notation for bit-wise symmetric signatures), let $g^{01}=1$ and $g^{10}=\Gamma^{10,01,01, \ldots, 01} / \Gamma^{01,01, \ldots, 01}=\Gamma^{10,01,01, \ldots, 01}$. From Claim 6, we can verify that $(11)$ is satisfied. This is seen as follows: Claim 6 allows one to "exchange" one block of 10 for one block of 01 , incurring a factor of $g^{10}$. This works as long as $g^{10} \neq 0$. If $g^{10}=0$, we can instead use Claim 6 to show that $\Gamma^{01,10, \alpha}=0$, for all $\alpha \in\{01,10\}^{n-2}$. Moreover we want to show that $\Gamma^{10,10, \ldots, 10}=0$ as well. For this purpose, we use MGI with the pattern $00,10,10, \ldots, 10$ and all positions, and get

$0=(10,10,10, \ldots, 10)(01,01,01, \ldots, 01)-(01,10,10, \ldots, 10)(10,01,01, \ldots, 01)+\ldots$

The remaining terms (omitted) all have a 00 block in its first factor, and so they are all 0 . The second term is also 0 as $g^{10}=0$. Yet $(01,01,01, \ldots, 01)=1$, so $(10,10,10, \ldots, 10)=0$. This proves the Claim when $\Gamma^{01,01, \ldots, 01} \neq 0$.

If $\Gamma^{01,01, \ldots, 01}=0$, again from the "exchange" argument by Claim 6 , the only possible non-zero entry of $\Gamma$ is $\Gamma^{10,10, \ldots, 10}$. Let $g^{00}=g^{11}=g^{01}=0$ and $g^{10}=\sqrt[n]{\Gamma^{10,10, \ldots, 10}}$. Then (11) is satisfied. (This may require us to go to an algebraic extension field.)

Claim 8 For $n \geq 4, k=2, n$ is odd and $\Gamma^{00,00, \ldots, 00}=\Gamma^{11,11, \ldots, 11}=0$, Theorem 2 holds.

Proof: Since $n$ is odd and $\Gamma$ is an even matchgate, from Claim 5, we know that if $\Gamma^{\alpha_{1} \alpha_{2} \cdots \alpha_{n}} \neq 0$, then there is exactly one $\alpha_{i} \in\{00,11\}$ and all other $\alpha_{j} \in\{01,10\}$. By block-symmetry, we assume $\alpha_{1} \in\{00,11\}$ and $\alpha_{i} \in\{01,10\}$ (where $i=2,3, \ldots, n$ ).

If $\Gamma^{00,01,01, \ldots, 01} \neq 0$, w.l.o.g, we assume $\Gamma^{00,01,01, \ldots, 01}=1$. Let $g^{00}=g^{01}=1$. Using the pattern $10,01,01, \ldots, 01$ and the first four bits as positions, we have

$$
(00,01)(11,10)-(11,01)(00,10)+(10,11)(01,00)-(10,00)(01,11)=0 .
$$

By block-symmetry, the first and the last two terms are equal. So we have

$$
\Gamma^{00,01,01, \ldots, 01} \Gamma^{11,10,01, \ldots, 01}-\Gamma^{11,01,01, \ldots, 01} \Gamma^{00,10,01, \ldots, 01}=0 .
$$

Since $\Gamma^{00,01,01, \ldots, 01}=1$, let $g^{11}=\Gamma^{11,01,01, \ldots, 01}$ and $g^{10}=\Gamma^{00,10,01, \ldots, 01}$, we have $\Gamma^{11,10,01, \ldots, 01}=g^{11} g^{10}$. And let $\Gamma_{s}$ be the matchgate with symmetric signature $[0,0, \ldots, 1,0]$. The proof is similar with Claim 7. Degenerate cases happen when $g^{10}=0$, or $g^{11}=0$, or both. In particular, when $g^{10}=0$, we need to prove $\Gamma^{00,10,10 \ldots 10}=0$, which goes beyond Claim 6 . This is shown by the MGI using the pattern $00,00,10, \ldots, 10$ and positions $00,11,11, \ldots, 11$ (all 
the bits except the first two). We also need to prove $\Gamma^{11,10,10, \ldots, 10}=0$ when $g^{10}=0$ or $g^{11}=0$ or both. This can be shown by the MGI using the pattern $10,01,01, \ldots, 01$ and all positions.

If $\Gamma^{11,01,01, \ldots, 01} \neq 0$, we have a similar proof.

Finally assume $\Gamma^{00,01,01, \ldots, 01}=\Gamma^{11,01,01, \ldots, 01}=0$. From Claim 6 and the "exchange" argument, the only two possible non-zero entries of $\Gamma$ are $\Gamma^{00,10,10, \ldots, 10}$ and $\Gamma^{11,10,10, \ldots, 10}$. If they are both 0 , then $\Gamma$ is trivial. Otherwise w.l.o.g. we assume $\Gamma^{00,10,10, \ldots, 10}=1$. Let $g^{01}=0, g^{00}=g^{10}=1$ and $g^{11}=\Gamma^{11,10,10, \ldots, 10}$. And let $\Gamma_{s}$ be the matchgate with symmetric signature $[0,0, \ldots, 1,0]$, we can verify that $(11)$ is satisfied.

Together with Claim 7 and Claim 8, we have a complete proof for Theorem 2.

This paper presents an elegant decomposition theorem on the structure of block-wise symmetric signatures for matchgates. The main tool is Matchgate Identities. However the statement of Theorem 2 for $k>2$ without any non-zero conditions is open. It would also be interesting to simplify the proofs.

\section{References}

1. J-Y. Cai and Vinay Choudhary. Some Results on Matchgates and Holographic Algorithms. In Proceedings of ICALP 2006, Part I. Lecture Notes in Computer Science vol. 4051. pp 703-714. Also available at ECCC TR06-048, 2006.

2. J-Y. Cai and Vinay Choudhary. On the Theory of Matchgate Computations. To appear in IEEE Conference on Computational Complexity 2007. Also available at ECCC Report TR06-018.

3. J-Y. Cai and Pinyan Lu. Bases Collapse in Holographic Algorithms. To appear in CCC 2007. Also available at ECCC Report TR07-003.

4. J. Edmonds. Minimum partition of a matroid into independent subsets. J. Res. Nat. Bur. Standards Sect. B, 69:67-72, 1965.

5. M. Jerrum. Two-dimensional monomer-dimer systems are computationally intractable. J. Stat. Phys. 48 (1987) 121-134; erratum, 59 (1990) 1087-1088

6. P. W. Kasteleyn. The statistics of dimers on a lattice. Physica, 27: 1209-1225 (1961).

7. P. W. Kasteleyn. Graph Theory and Crystal Physics. In Graph Theory and Theoretical Physics, (F. Harary, ed.), Academic Press, London, 43-110 (1967).

8. K. Murota. Matrices and Matroids for Systems Analysis, Springer, Berlin, 2000.

9. H. N. V. Temperley and M. E. Fisher. Dimer problem in statistical mechanics an exact result. Philosophical Magazine 6: 1061- 1063 (1961).

10. L. G. Valiant. Quantum circuits that can be simulated classically in polynomial time. SIAM Journal of Computing, 31(4): 1229-1254 (2002).

11. L. G. Valiant. Expressiveness of Matchgates. Theoretical Computer Science, 281(1): 457-471 (2002).

12. L. G. Valiant. Holographic Algorithms (Extended Abstract). In Proc. 45th IEEE Symposium on Foundations of Computer Science, 2004, 306-315. A more detailed version appeared in ECCC Report TR05-099.

13. L. G. Valiant. Accidental Algorithms. In Proc. 47th Annual IEEE Symposium on Foundations of Computer Science 2006, 509-517. 


\section{Appendix A: Decomposition Theory for Odd Matchgate}

Theorem 3. Let $\left(\Gamma^{\alpha}\right)$ be a block-wise symmetric tensor with block size $k$ and arity $n k$. Assume $n \geq 4$ and $\Gamma^{e_{1} 0 \cdots 0} \neq 0$. Then $\Gamma$ is realizable by a matchgate iff there exist a matchgate $\Gamma_{0}$ with arity $k+1$ and condensed signature $\left(g^{\alpha}\right)_{\alpha \in\{0,1\}^{k}}$, and a symmetric matchgate $\Gamma_{s}$ such that

$$
\Gamma^{\alpha_{1} \alpha_{2} \cdots \alpha_{n}}=\Gamma_{s}^{p\left(\alpha_{1}\right) p\left(\alpha_{2}\right) \cdots p\left(\alpha_{n}\right)} g^{\alpha_{1}} g^{\alpha_{2}} \cdots g^{\alpha_{n}}
$$

Proof: " $\Leftarrow "$ can be proved in the same way as in Theorem 1 .

Now we prove " $\Rightarrow "$ : Since $\Gamma^{100 \cdots 0} \neq 0$, w.l.o.g, we can assume $\Gamma^{100 \cdots 0}=1$.

Let $r_{1}=\Gamma^{e_{1} e_{1} e_{1} 00 \cdots 0} \neq 0$. We take $\Gamma_{s}$ to be an odd symmetric matchgate with signature $z_{2 i+1}=\left(r_{1}\right)^{i}$. By [2] this $\Gamma_{s}$ exists. Since the given $\left(\Gamma^{\alpha}\right)$ is realizable, it can be realized by a matchgate $\Gamma$ with $n k$ external nodes. View its first $k+1$ external nodes still as external nodes and other nodes as internal, we have a matchgate with $k+1$ external nodes. This is our $\Gamma_{0}$. By definition its condensed signature is

$$
g^{\alpha}=\left\{\begin{aligned}
\Gamma^{\alpha 00 \cdots 0} & \text { when } \operatorname{wt}(\alpha) \text { is odd } \\
\Gamma^{\alpha e_{1} 0 \cdots 0} & \text { when } \operatorname{wt}(\alpha) \text { is even. }
\end{aligned}\right.
$$

Note that in this definition $g^{\alpha}=1$ for both $\alpha=0^{k}$ and $\alpha=e_{1} \in\{0,1\}^{k}$.

We prove (13) by induction on $\operatorname{wt}\left(\alpha_{1} \alpha_{2} \cdots \alpha_{n}\right) \geq 0$ and $\operatorname{wt}\left(\alpha_{1} \alpha_{2} \cdots \alpha_{n}\right)$ is odd.

The base case $\operatorname{wt}\left(\alpha_{1} \alpha_{2} \cdots \alpha_{n}\right)=1$ is obvious. However before we deal with the case $\operatorname{wt}\left(\alpha_{1} \alpha_{2} \cdots \alpha_{n}\right)=3$, we first establish some identities.

Using the pattern and positions both $e_{i} e_{j} e_{1} e_{1} 00 \cdots 0$ (for arbitrary $i, j \in[k]$ ), we have MGI:

$\Gamma^{e_{j} e_{1} e_{1} 0 \cdots 0} \Gamma^{e_{i} 0 \cdots 0}-\Gamma^{e_{i} e_{1} e_{1} 0 \cdots 0} \Gamma^{e_{j} 0 \cdots 0}+\Gamma^{e_{i} e_{j} e_{1} 0 \cdots 0} \Gamma^{e_{1} 0 \cdots 0}-\Gamma^{e_{i} e_{j} e_{1} 0 \cdots 0} \Gamma^{e_{1} 0 \cdots 0}=0$.

The last two terms cancel out, and we get:

$$
\Gamma^{e_{j} e_{1} e_{1} 00 \cdots 0} \Gamma^{e_{i} 00 \cdots 0}=\Gamma^{e_{i} e_{1} e_{1} 00 \cdots 0} \Gamma^{e_{j} 00 \cdots 0} .
$$

Next, using the pattern and position both $e_{i} e_{1} e_{j} e_{1} 00 \cdots 0$, we have the following matchgate identity:

$\Gamma^{e_{j} e_{1} e_{1} 0 \cdots 0} \Gamma^{e_{i} 0 \cdots 0}-\Gamma^{e_{i} e_{1} e_{j} 0 \cdots 0} \Gamma^{e_{1} 0 \cdots 0}+\Gamma^{e_{i} e_{1} e_{1} 0 \cdots 0} \Gamma^{e_{j} 0 \cdots 0}-\Gamma^{e_{i} e_{1} e_{j} 0 \cdots 0} \Gamma^{e_{1} 0 \cdots 0}=0$.

Together with (14), we have:

$$
\Gamma^{e_{i} e_{j} e_{1} 00 \cdots 0} \Gamma^{e_{1} 00 \cdots 0}=\Gamma^{e_{1} e_{1} e_{j} 00 \cdots 0} \Gamma^{e_{i} 00 \cdots 0}=\Gamma^{e_{1} e_{1} e_{i} 00 \cdots 0} \Gamma^{e_{j} 00 \cdots 0}
$$

Let $j=1$ in the above equation and note that $\Gamma^{e_{1} 00 \cdots 0}=1$ and $\Gamma^{e_{1} e_{1} e_{1} \cdots 0}=$ $r_{1}$, we have

$$
\Gamma^{e_{i} e_{1} e_{1} 00 \cdots 0}=\Gamma^{e_{1} e_{1} e_{1} 00 \cdots 0} \Gamma^{e_{i} 00 \cdots 0}=r_{1} g^{e_{i}} .
$$

Substituting this in (15), we have: 


$$
\Gamma^{e_{i} e_{j} e_{1} 00 \cdots 0}=r_{1} g^{e_{i}} g^{e_{j}} .
$$

Now we come back to (part of the inductive base case) where $\operatorname{wt}\left(\alpha_{1} \alpha_{2} \cdots \alpha_{n}\right)=$ 3 . We have three cases: the three 1s are in 1 block, 2 blocks or 3 blocks.

The case that they are in the same block is obvious by definition.

Next we consider the other two cases. If three 1s are in two blocks, then it has the form $\Gamma^{e_{i}\left(e_{j}+e_{l}\right) 00 \cdots 0}(j \neq l)$. Using the pattern and positions both $e_{1} e_{i}\left(e_{j}+e_{l}\right) 00 \cdots 0$, we have MGI:

$\Gamma^{e_{i}\left(e_{j}+e_{l}\right) 0 \cdots 0} \Gamma^{e_{1} 0 \cdots 0}-\Gamma^{e_{1}\left(e_{j}+e_{l}\right) 0 \cdots 0} \Gamma^{e_{i} 0 \cdots 0}+\Gamma^{e_{1} e_{i} e_{l} 0 \cdots 0} \Gamma^{e_{j} 0 \cdots 0}-\Gamma^{e_{1} e_{i} e_{j} 0 \cdots 0} \Gamma^{e_{l} 0 \cdots 0}=0$.

Substituting (16) in the above equation, we find the last two terms cancel out. And by definition, $\Gamma^{e_{1}\left(e_{j}+e_{l}\right) 00 \cdots 0}=g^{\left(e_{j}+e_{l}\right)}$. Therefore, we have

$$
\Gamma^{e_{i}\left(e_{j}+e_{l}\right) 00 \cdots 0}=g^{e_{i}} g^{\left(e_{j}+e_{l}\right)} .
$$

This satisfies (13).

The last case is that three $1 \mathrm{~s}$ are in three blocks. Then it has the form $\Gamma^{e_{i} e_{j} e_{l} 00 \cdots 0}$. Using the pattern and positions both $e_{1} e_{i} e_{j} e_{l} 00 \cdots 0$, we have MGI:

$\Gamma^{e_{i} e_{j} e_{l} 0 \cdots 0} \Gamma^{e_{1} 0 \cdots 0}-\Gamma^{e_{1} e_{j} e_{l} 0 \cdots 0} \Gamma^{e_{i} 0 \cdots 0}+\Gamma^{e_{1} e_{i} e_{l} 0 \cdots 0} \Gamma^{e_{j} 0 \cdots 0}-\Gamma^{e_{1} e_{i} e_{j} 0 \cdots 0} \Gamma^{e_{l} 0 \cdots 0}=0$.

Substituting (16) in it, we find the last two terms cancel out and

$$
\Gamma^{e_{i} e_{j} e_{l} 00 \cdots 0}=r_{1} g^{e_{i}} g^{e_{j}} g^{e_{l}} .
$$

This also satisfies (13).

Inductively we assume (13) has been proved for all $\operatorname{wt}\left(\alpha_{1} \alpha_{2} \cdots \alpha_{n}\right) \leq 2(i-$ $1)+1$, for some $i \geq 2$. Now $\operatorname{wt}\left(\alpha_{1} \alpha_{2} \cdots \alpha_{n}\right)=2 i+1 \geq 5$.

By symmetry, we can assume $\alpha_{1} \neq 0^{k}$. Consider the first bit of $\alpha_{1}$, there are two cases: it is 1 or 0 .

First we assume the first bit of $\alpha_{1}$ is 1 . Using positions $\left(\alpha_{1}+e_{1}\right) \alpha_{2} \cdots \alpha_{n}$ and the pattern $\alpha_{1} \alpha_{2} \cdots \alpha_{n}+e_{t}$, where $t$ is the position of the first 1 in the pattern $\left(\alpha_{1}+e_{1}\right) \alpha_{2} \cdots \alpha_{n}$, we have MGI:

$$
\Gamma^{\alpha_{1} \alpha_{2} \cdots \alpha_{n}}=\sum_{j=2}^{2 i}(-1)^{j} \Gamma^{\alpha_{1} \alpha_{2} \cdots \alpha_{n}+e_{t}+e_{p_{j}}} \Gamma^{e_{1}+e_{t}+e_{p_{j}}} .
$$

Note that every $\Gamma^{\beta}$ in the RHS has weight $\operatorname{wt}(\beta) \leq 2 i-1$, so we can apply (13) to them. Again we do the summation block by block; the sum of the $r$-th block is denoted as $S_{r}$. Let $2 q+1$ be the number of blocks with odd weight in pattern $\alpha_{1} \alpha_{2} \cdots \alpha_{n}$. Note that this number is odd.

Now we must divide the proof into two cases, depending on whether $t$ is in the first block $\left(\alpha_{1}+e_{1}\right)$ or not. If it is not, then $\alpha_{1}=e_{1}$. In this case the first block is not involved in the MGI at all. Exactly the same proof as in Theorem 1 works here. 
So we assume $t$ is in the first block $\left(\alpha_{1}+e_{1}\right)$. For the first block, let $w_{1}=$ $\operatorname{wt}\left(\alpha_{1}+e_{1}\right)=\operatorname{wt}\left(\alpha_{1}\right)-1$. If $w_{1}=1$, then $S_{1}=0$, being an empty sum. Assume $w_{1}>1$. In the notation below we consider $e_{t}, e_{p_{j}} \in\{0,1\}^{k}$ for convenience.

$$
\begin{aligned}
S_{1} & =\sum_{j=2}^{w_{1}}(-1)^{j} \Gamma^{\left(\alpha_{1}+e_{t}+e_{p_{j}}\right) \alpha_{2} \cdots \alpha_{n}} \Gamma^{\left(e_{1}+e_{t}+e_{p_{j}}\right) 00 \cdots 0} \\
& =r_{1}^{q} g^{\alpha_{2}} \cdots g^{\alpha_{n}} \sum_{j=2}^{w_{1}}(-1)^{j} g^{\alpha_{1}+e_{t}+e_{p_{j}}} g^{e_{1}+e_{t}+e_{p_{j}}} .
\end{aligned}
$$

Note that the exponent $q$ in $r_{1}^{q}$ comes from the fact that the number of blocks with odd weight among $\alpha_{1}+e_{t}+e_{p_{j}}, \alpha_{2}, \ldots, \alpha_{n}$ is $2 q+1$.

If $w_{1}$ is odd, using the pattern $\left(\alpha_{1}+e_{t}\right) 1$ and positions $\left(\alpha_{1}+e_{1}\right) 1$, we have the following MGI for $\Gamma_{0}$ :

$$
-g^{\alpha_{1}}+\sum_{j=2}^{w_{1}}(-1)^{j} g^{\alpha_{1}+e_{t}+e_{p_{j}}} g^{e_{1}+e_{t}+e_{p_{j}}}+g^{\alpha_{1}+e_{t}} g^{e_{t}+e_{1}}=0 .
$$

Substituting this in (19), we have:

$$
S_{1}=r_{1}^{q} g^{\alpha_{2}} \cdots g^{\alpha_{n}}\left(g^{\alpha_{1}}-g^{\alpha_{1}+e_{t}} g^{e_{t}+e_{1}}\right) .
$$

We note that this is also valid for $w_{1}=1$.

If $w_{1}$ is even, using the pattern $\left(\alpha_{1}+e_{t}\right) 0$ and positions $\left(\alpha_{1}+e_{1}\right) 0$, we have the following matchgate identity for $\Gamma_{0}$ :

$$
-g^{\alpha_{1}}+\sum_{j=2}^{w_{1}}(-1)^{j} g^{\alpha_{1}+e_{t}+e_{p_{j}}} g^{e_{1}+e_{t}+e_{p_{j}}}=0 .
$$

Substituting this in (19), we have:

$$
S_{1}=r_{1}^{q} g^{\alpha_{1}} g^{\alpha_{2}} \cdots g^{\alpha_{n}} .
$$

If all $S_{r}$ are empty block-wise sums for $r>1$ (i.e., $w_{r}=0$ for all $r>1$ ), then $w_{1}$ must be even (this means $\operatorname{wt}\left(\alpha_{1}\right)$ is odd), and we are done. Now suppose there are non-empty block-wise sums $S_{r}$, for $r>1$. For the $r$-th block, let $w_{r}=\operatorname{wt}\left(\alpha_{r}\right)$ and $v_{r}$ be the number of $1 \mathrm{~s}$ in the first $r-1$ blocks of the pattern $\left(\alpha_{1}+e_{1}\right) \alpha_{2} \cdots \alpha_{n}$, and $p_{j}^{r}$ (where $j \in\left[w_{r}\right]$ ) be the position of the $j$-th 1 in $\alpha_{r}$. We have

$$
\begin{aligned}
S_{r} & =(-1)^{v_{r}} \sum_{j=1}^{w_{r}}(-1)^{j} \Gamma^{\left(\alpha_{1}+e_{t}\right) \alpha_{2} \cdots\left(\alpha_{r}+e_{p_{j}^{r}}\right) \cdots \alpha_{n}} \Gamma^{\left(e_{1}+e_{t}\right) 00 \cdots\left(e_{p_{j}^{r}}\right) \cdots 0} \\
& =(-1)^{v_{r}} r_{1}^{q^{\prime}} g^{e_{1}+e_{t}} g^{\alpha_{1}+e_{t}} g^{\alpha_{2}} \cdots \widehat{g^{\alpha_{r}}} \cdots g^{\alpha_{n}} \sum_{j=1}^{w_{r}}(-1)^{j} g^{\alpha_{r}+e_{p_{j}^{r}}} g^{e_{p_{j}^{r}}}
\end{aligned}
$$

where $\widehat{g^{\alpha_{r}}}$ denotes a missing factor, and $2 q^{\prime}+1$ is the total number of odd blocks in $\alpha_{1}+e_{t}, \alpha_{2}, \ldots, \alpha_{r}+e_{p_{j}^{r}}, \ldots, \alpha_{n}$. 
If $w_{r}$ is even, using the pattern and positions to be both $\alpha_{r} 0$, we have the following MGI for $\Gamma_{0}$ :

$$
\sum_{j=1}^{w_{r}}(-1)^{j} g^{\alpha_{r}+e_{p_{j}^{r}}} g^{e_{p_{j}^{r}}}=0 .
$$

Substituting this in (20) and (21), we have $S_{r}=0$.

Therefore, among block sums $S_{r}$, for $r>1$, we need only consider blocks with odd $w_{r}$. Assume $w_{r}$ is odd now, we have $q^{\prime}=q$ if $w_{1}$ is odd, and $q^{\prime}=q-1$ if $w_{1}$ is even. Using the pattern and positions to be both $\alpha_{r} 1$, we have the following MGI for $\Gamma_{0}$ :

$$
\sum_{j=1}^{w_{r}}(-1)^{j} g^{\alpha_{r}+e_{p_{j}^{r}}} g^{e_{p_{j}^{r}}}+g^{\alpha_{r}}=0 .
$$

Substituting this in (20) and (21), we have

$$
S_{r}=-(-1)^{v_{r}} r_{1}^{q^{\prime}} g^{e_{t}+e_{1}} g^{\alpha_{1}+e_{t}} g^{\alpha_{2}} \cdots g^{\alpha_{r}} \cdots g^{\alpha_{n}} .
$$

To sum up, after the first block sum $S_{1}$, every even block will be zero, and every odd block will alternatingly contribute a $\pm r_{1}^{q^{\prime}} g^{e_{t}+e_{1}} g^{\alpha_{1}+e_{t}} g^{\alpha_{2}} \cdots g^{\alpha_{n}}$. If $S_{1}$ is an even block sum (this means wt $\left(\alpha_{1}+e_{1}\right)$ is even, but wt $\left(\alpha_{1}\right)$ is odd), then this alternating sum has an even number of such terms, and they all cancel out. This leaves us with the desired result $\Gamma^{\alpha_{1} \alpha_{2} \cdots \alpha_{n}}=S_{1}=r_{1}^{q} g^{\alpha_{1}} g^{\alpha_{2}} \cdots g^{\alpha_{n}}$.

If $S_{1}$ is an odd block sum (this means wt $\left(\alpha_{1}+e_{1}\right)$ is odd, but $\operatorname{wt}\left(\alpha_{1}\right)$ is even), then $q^{\prime}=q$, and there are an odd number of alternating terms from $S_{r}$ for $r>1$, starting with the sign $-(-1)^{v_{2}}=+1$. (Note that $v_{2}=w_{1}=\operatorname{wt}\left(\alpha_{1}+e_{1}\right)$ is odd.) These will cancel out pairwise except one $r_{1}^{q} g^{e_{t}+e_{1}} g^{\alpha_{1}+e_{t}} g^{\alpha_{2}} \cdots g^{\alpha_{n}}$ left, which cancels the $-r_{1}^{q} g^{e_{t}+e_{1}} g^{\alpha_{1}+e_{t}} g^{\alpha_{2}} \cdots g^{\alpha_{n}}$ in $S_{1}$. In either cases, we have

$$
\Gamma^{\alpha_{1} \alpha_{2} \cdots \alpha_{n}}=r_{1}^{q} g^{\alpha_{1}} g^{\alpha_{2}} \cdots g^{\alpha_{n}} .
$$

Now we come back to the other case where the first bit of $\alpha_{1}$ is 0 .

Using the pattern and positions both $\left(\alpha_{1}+e_{1}\right) \alpha_{2} \cdots \alpha_{n}$, we have MGI:

$$
\Gamma^{\alpha_{1} \alpha_{2} \cdots \alpha_{n}}=\sum_{j=2}^{2 i}(-1)^{j} \Gamma^{\left(\alpha_{1}+e_{1}\right) \alpha_{2} \cdots \alpha_{n}+e_{p_{j}}} \Gamma^{e_{p_{j}}} .
$$

Note that $\operatorname{wt}\left(\left(\alpha_{1}+e_{1}\right) \alpha_{2} \cdots \alpha_{n}+e_{p_{j}}\right)=\operatorname{wt}\left(\alpha_{1} \alpha_{2} \cdots \alpha_{n}\right)=2 i+1$ in the RHS, but the first bit of $\left(\alpha_{1}+e_{1}\right) \alpha_{2} \cdots \alpha_{n}+e_{p_{j}}$ is 1 . For these indices, we have already proved that (13) is satisfied.

Therefore we can apply (13) in the RHS of (22) and do the summation block by block. For the first block, let $w_{1}=\operatorname{wt}\left(\alpha_{1}+e_{1}\right)\left(=\operatorname{wt}\left(\alpha_{1}\right)+1\right)$. Note that $w_{1}>1$ since we assumed $\alpha_{1} \neq 0^{k}$.

$S_{1}=\sum_{j=2}^{w_{1}}(-1)^{j} \Gamma^{\left(\alpha_{1}+e_{1}+e_{p_{j}}\right) \alpha_{2} \cdots \alpha_{n}} \Gamma^{e_{p_{j}} 0 \cdots 0}=r_{1}^{q} g^{\alpha_{2}} \cdots g^{\alpha_{n}} \sum_{j=2}^{w_{1}}(-1)^{j} g^{\alpha_{1}+e_{1}+e_{p_{j}}} g^{e_{p_{j}}}$. 
Note that the exponent $q$ in $r_{1}^{q}$ comes from the fact that the number of blocks with odd weight among $\alpha_{1}+e_{1}+e_{p_{j}}, \alpha_{2}, \ldots, \alpha_{n}$ is $2 q+1$.

If $w_{1}$ is odd, using the pattern and positions both $\left(\alpha_{1}+e_{1}\right) 1$, we have the following MGI for $\Gamma_{0}$ :

$$
-g^{\alpha_{1}}+\sum_{j=2}^{w_{1}}(-1)^{j} g^{\alpha_{1}+e_{1}+e_{p_{j}}} g^{e_{p_{j}}}+g^{\alpha_{1}+e_{1}}=0 .
$$

Here we used $g^{e_{1}}=g^{0^{k}}=1$. Substituting this in (23), we have:

$$
S_{1}=r_{1}^{q} g^{\alpha_{2}} \cdots g^{\alpha_{n}}\left(g^{\alpha_{1}}-g^{\alpha_{1}+e_{1}}\right) .
$$

If $w_{1}$ is even, using the pattern and positions both $\left(\alpha_{1}+e_{1}\right) 0$, we have the following MGI for $\Gamma_{0}$ :

$$
-g^{\alpha_{1}}+\sum_{j=2}^{w_{1}}(-1)^{j} g^{\alpha_{1}+e_{1}+e_{p_{j}}} g^{e_{p_{j}}}=0 .
$$

Substituting this in (23), we have:

$$
S_{1}=r_{1}^{q} g^{\alpha_{1}} g^{\alpha_{2}} \cdots g^{\alpha_{n}} .
$$

If all $S_{r}$ are empty block-wise sums for $r>1$ (i.e., $w_{r}=0$ for all $r>1$ ), then $w_{1}$ must be even, and we are done. Now suppose there are non-empty block-wise sums $S_{r}$, for $r>1$. For the $r$-th block, let $w_{r}=\operatorname{wt}\left(\alpha_{r}\right)$ and $v_{r}$ be the number of $1 \mathrm{~s}$ in the first $r-1$ blocks of the pattern $\left(\alpha_{1}+e_{1}\right) \alpha_{2} \cdots \alpha_{n}$, and $p_{j}^{r}$ (where $\left.j \in\left[w_{r}\right]\right)$ be the position of the $j$-th 1 in $\alpha_{r}$. We have

$$
\begin{aligned}
S_{r} & =(-1)^{v_{r}} \sum_{j=1}^{w_{r}}(-1)^{j} \Gamma^{\left(\alpha_{1}+e_{1}\right) \alpha_{2} \cdots\left(\alpha_{r}+e_{p_{j}^{r}}\right) \cdots \alpha_{n}} \Gamma^{000 \cdots\left(e_{p_{j}^{r}}\right) \cdots 0} \\
& =(-1)^{v_{r}} r_{1}^{q^{\prime}} g^{\alpha_{1}+e_{1}} g^{\alpha_{2}} \cdots \widehat{g^{\alpha_{r}}} \cdots g^{\alpha_{n}} \sum_{j=1}^{w_{r}}(-1)^{j} g^{\alpha_{r}+e_{p_{j}^{r}}} g^{e_{p_{j}^{r}}}
\end{aligned}
$$

where $\widehat{g^{\alpha_{r}}}$ denotes a missing factor, and $2 q^{\prime}+1$ is the total number of odd blocks in $\alpha_{1}+e_{1}, \alpha_{2}, \ldots, \alpha_{r}+e_{p_{j}^{r}}, \ldots, \alpha_{n}$. We also used $g^{0^{k}}=1$.

If $w_{r}$ is even, using the pattern and positions both $\alpha_{r} 0$, we have the following MGI for $\Gamma_{0}$ :

$$
\sum_{j=1}^{w_{r}}(-1)^{j} g^{\alpha_{r}+e_{p_{j}^{r}}} g^{e_{p_{j}^{r}}^{r}}=0 .
$$

Substituting this in (24) and (25), we have $S_{r}=0$.

Therefore, among block sums $S_{r}$, for $r>1$, we need only consider blocks with odd $w_{r}$. Assume $w_{r}$ is odd now, we have $q^{\prime}=q$ if $w_{1}$ is odd, and $q^{\prime}=q-1$ if $w_{1}$ is even. Using the pattern and positions both $\alpha_{r} 1$, we have the following MGI for $\Gamma_{0}$ :

$$
\sum_{j=1}^{w_{r}}(-1)^{j} g^{\alpha_{r}+e_{p_{j}^{r}}} g^{e_{p_{j}^{r}}}+g^{\alpha_{r}}=0 .
$$


Substituting this in (24) and (25), we have

$$
S_{r}=-(-1)^{v_{r}} r_{1}^{q^{\prime}} g^{\alpha_{1}+e_{1}} g^{\alpha_{2}} \cdots g^{\alpha_{r}} \cdots g^{\alpha_{n}} .
$$

To sum up, after the first block sum $S_{1}$, every even block will be zero, and every odd block will alternatingly contribute a $\pm r_{1}^{q^{\prime}} g^{\alpha_{1}+e_{t}} g^{\alpha_{2}} \cdots g^{\alpha_{n}}$. If $S_{1}$ is an even block sum (this means wt $\left(\alpha_{1}+e_{1}\right)$ is even), then this alternating sum has an even number of such terms, and they all cancel out. This leaves us with the desired result $\Gamma^{\alpha_{1} \alpha_{2} \cdots \alpha_{n}}=S_{1}=r_{1}^{q} g^{\alpha_{1}} g^{\alpha_{2}} \cdots g^{\alpha_{n}}$.

If $S_{1}$ is an odd block sum (this means wt $\left(\alpha_{1}+e_{1}\right)$ is odd), then $q^{\prime}=q$, and there are an odd number of alternating terms from $S_{r}$ for $r>1$, starting with the $\operatorname{sign}-(-1)^{v_{2}}=+1$. These will cancel out pairwise except one $r_{1}^{q} g^{\alpha_{1}+e_{1}} g^{\alpha_{2}} \cdots g^{\alpha_{n}}$ left, which cancels the $-r_{1}^{q} g^{\alpha_{1}+e_{1}} g^{\alpha_{2}} \cdots g^{\alpha_{n}}$ in $S_{1}$. In either cases, we have finally

$$
\Gamma^{\alpha_{1} \alpha_{2} \cdots \alpha_{n}}=r_{1}^{q} g^{\alpha_{1}} g^{\alpha_{2}} \cdots g^{\alpha_{n}} .
$$

\section{Appendix B: Proof of Claim 5}

Proof: For any $\alpha^{\prime} \in\{00,01,10,11\}^{n-4}$, using the pattern 10,10,10,11, $\alpha^{\prime}$ and positions 10,10,01,01, we have MGI:

$$
\begin{array}{r}
0=(00,10,10,11)(10,00,11,10) \\
-(10,00,10,11)(00,10,11,10) \\
+(10,10,11,11)(00,00,10,10) \\
-(10,10,10,10)(00,00,11,11)
\end{array}
$$

Since the first two terms cancel and from Claim 4 the third term is 0 , we have

$$
(10,10,10,10)(00,00,11,11)=0 .
$$

Using the pattern $10,10,10,11, \alpha^{\prime}$ and positions $10,01,10,01$, we have MGI (here in all displayed entries of signature $\Gamma$ in MGI we omit $\Gamma$ and $\alpha^{\prime}$ and display only the first 8 bits):

$$
\begin{array}{r}
0=(00,10,10,11)(10,11,00,10) \\
-(10,11,10,11)(00,10,00,10) \\
+(10,10,00,11)(00,11,10,10) \\
-(10,10,10,10)(00,11,00,11)
\end{array}
$$

From Claim 4 we know the second term is 0 and from (26) we know the last term is 0 , and since the first and the third terms are the same, we have

$$
(00,10,10,11)=0 .
$$


Similarly, we have

$$
(00,01,01,11)=0 .
$$

Using the pattern $10,10,01,11, \alpha^{\prime}$ and positions $10,10,10,10$, we have MGI:

$$
\begin{array}{r}
0=(00,10,01,11)(10,00,11,01) \\
-(10,00,01,11)(00,10,11,01) \\
+(10,10,11,11)(00,00,01,01) \\
-(10,10,01,01)(00,00,11,11)
\end{array}
$$

Since the first two terms cancel and the third term is 0 by Claim 4 , we have

$$
(10,10,01,01)(00,00,11,11)=0 .
$$

Using the pattern $10,01,10,11, \alpha^{\prime}$ and positions $10,10,10,10$, we have MGI:

$$
\begin{array}{r}
0=(00,01,10,11)(10,11,00,01) \\
-(10,11,10,11)(00,01,00,01) \\
+(10,01,00,11)(00,11,10,01) \\
-(10,01,10,01)(00,11,00,11)
\end{array}
$$

From Claim 4 we know the second term is 0 and from (29) we know the last term is 0 , since the first and the third terms are the same, we have

$$
(10,01,00,11)=0 .
$$

Using the pattern $00,01,00,11, \alpha^{\prime}$ and positions $11,11,00,00$, we have MGI:

$$
\begin{array}{r}
0=(10,01,00,11)(01,10,00,11) \\
-(01,01,00,11)(10,10,00,11) \\
+(00,11,00,11)(11,00,00,11) \\
-(00,00,00,11)(11,11,00,11)
\end{array}
$$

From Claim 3 we know that the last term is 0 and from (30) and (27) we know that the first two terms are 0 . So we have

$$
(11,00,00,11)=0 .
$$

Now finally we are ready to prove Claim 5 . We first prove $G^{00,00, \alpha}=0$.

If $\alpha$ has any block 00, from Claim 3, we have $G^{00,00, \alpha}=0$.

If $\alpha$ has any block of weight 1 , then there must be at least two blocks of weight 1 . So from Claim 4, we have $G^{00,00, \alpha}=0$.

Otherwise every block of $\alpha$ is 11 . Then from (31), we know $G^{00,00, \alpha}=0$.

$G^{11,11, \alpha}=0$ can be proved similarly.

Now we prove $G^{00,11, \alpha}=0$. If $\alpha$ contains any block of 00 or 11 , it has been proved. Otherwise, every block of $\alpha$ is 01 or 10. Then from (27), (28) and (30) we have $G^{00,11, \alpha}=0$. 